\title{
Chicken of the Woods Laetiporus Sulphureus and Schizophyllum Commune Treasure of Medicinal Mushrooms
}

\author{
Elkhateeb WA ${ }^{1} *$, El Ghwas DE ${ }^{1}$, Gundoju NR ${ }^{2}$, Somasekhar T ${ }^{3}$, Akram $\mathbf{M}^{4}$ \\ and Daba GM1 ${ }^{1}$ \\ ${ }^{1}$ Chemistry of Natural and Microbial Products Department, National Research Centre, Egypt \\ ${ }^{2}$ Department of Chemistry, MR Degree College, India \\ ${ }^{3}$ Department of Chemistry, SVRM College Nagaram, India \\ ${ }^{4}$ Department of Eastern Medicine, Government College University Faisalabad, Pakistan
}

\section{Review Article \\ Volume 6 Issue 3}

Received Date: July 05, 2021

Published Date: July 28, 2021

DOI: $10.23880 /$ oajmb-16000201

*Corresponding author: Waill A Elkhateeb, Chemistry of Natural and Microbial Products Department, Pharmaceutical Industries Researches Division, National Research Centre, El Buhouth St, Dokki, 12311, Giza, Egypt, Email: waillahmed@yahoo.com

\section{Abstract}

The medicinal uses of the mushroom still need to be worked out for their biological activities. Mushrooms are small pharmaceutical factories, manufacturing various promising biologically active chemical compounds. These compounds exist in the mushroom fruit bodies, cultured mycelium, and culture broth. The presence of various phenolic compounds, polysaccharides, terpenoids, $\beta$-glucans, schizophyllan, ganoderic acid, and other compounds, is the reason for their potent biological activities, much more biological activities are discovered every day. Several compounds are responsible for the therapeutic activities of many medicinal mushrooms genera; the main groups of these compounds are polysaccharides, terpenes, phenolic compounds, and essential amino acids, as well as minerals such as such as calcium, potassium, magnesium, iron, and zinc. Overall, studies both on the chemistry and pharmacology of Laetiporus sulphureus and Schizophyllum commune extracts and compounds are increasing in recent years and show therapeutic potential for various pathologies. The purpose of this review was to investigate the biological activities of extract prepared from fruiting bodies of Laetiporus sulphureus and Schizophyllum commune.

Keywords: Medicinal Mushrooms; Laetiporus Sulphureus; Schizophyllum Commune; Biological Activities

Abbreviations: H-SMPS: Hot-Water Extractable Polysaccharides; E-SMPS: Enzymatic-Extractable Polysaccharides; DPPH: Diphenyl-1-Picrylhydrazyl; MICs: Minimum Inhibitory Concentrations.

\section{Introduction}

Mushrooms have been used as a traditional medicine for ages. Mushrooms as higher Basidiomycetes and Ascomycetes contain secondary metabolites in their fruit bodies, cultured mycelium, and cultured broth. Mushrooms have been used in many sides of human activity for many years [1]. Mushrooms are consumed in many countries as a cooking delicacy, particularly for their taste. In recent years, scientists have expanded their research area concerning other uses of mushrooms, particularly for medicinal and food-preserving purposes. Some of these mushrooms have been called medicinal mushrooms due to their various morphological, physiological, and ecological characteristics that are also responsible for their diversity. The mushrooms constitute 16,000 species worldwide with more than 2000 species identified as safe. 


\section{Open Access Journal of Microbiology \& Biotechnology}

Among these mushrooms, 1000 are edible, while others have been used as a source of biofuel, in medicinal formulation, as biochemicals, and for other purposes. Mushrooms have also huge potential, such as a "mushroom pharmaceuticals" with 130 medicinal functions [2]. Medicinal mushrooms possess medicinal properties such as anti-tumor, immunomodulating, antioxidant, cardiovascular, anti-hypercholesterolemic, antiviral, anti-bacterial, anti-parasitic, antifungal, detoxification, hepatoprotective, and anti-diabetic effects [3-9].

Mushrooms have been reported as the most valuable microbes to humankind $[10,11]$, and have been valued throughout the world both as food and medicine, mushrooms possess high contents of qualitative protein, crude fibre, minerals and vitamins. Investigations on the therapeutic and nutritional properties of mushrooms are on-going throughout the world. Researchers are providing vital data on the collection of biologically active secondary metabolites originated from mushrooms. Generally, mushrooms grow wild in many environments around the world and are also commercially cultivated for pharmaceutical and nutritional purposes. Nutritionally, mushrooms are healthy food which is rich in nutrients and vitamins. On the other hand, mushrooms have pharmaceutical and medical applications from centuries especially in Asian countries [12-14]. Mushrooms have been exploited in the last years as an alternative source of new antimicrobials. Wild and cultivated mushrooms contain a huge diversity of biomolecules with nutritional and/or medicinal properties. Because of their medicinal properties, mushrooms traditionally could be used with great potential for therapeutic applications in the treatment of some diseases. Life-threaten diseases, especially those caused by viruses, require searching and investigation in order to find potent compounds and drugs. Mushrooms are promising sources of compounds showing bioactive potency whenever tested. Recently, isolation and purification of biologically active secondary metabolites from mushrooms has been showed mainly on the products of fruiting bodies. Though, collection of fruiting bodies is a difficult work and is limited by collecting season and area. Also the small amounts of the collected samples are limited to find metabolites from mushrooms. Thus, mycelial culture of basidiomycetes and ascomycetes originated from wild mushrooms would be suggested as an alternative, dependable, and controllable method that can obtain various bioactive metabolites from mushrooms [15-20].

\section{Laetiporus Sulphureus}

Laetiporus sulphureus (Bull.) Murrill (also known as sulfur polypore, sulfur shelf, or chicken of the woods) is an edible wood-rotting basidiomycete fungus belonging to the family Polyporaceae. It is widely distributed in Asia, Europe, and North America. This mushroom commonly occurs on hardwoods or conifers [21-23]. Laetiporus sulphureus can be easily recognized due to its striking yellowish or orangecoloured shelf-like fruit bodies. Also is easily recognizable in forests and urban areas due to its impressive size (up to $40 \mathrm{~cm}$ wide) and vibrant bright sulphur yellow to orange coloured porous basidiocarps (Figures 1 \& 2). Laetiporus sulphureus is a source of bioactive compounds such as mono- and polysaccharides, laetiporic acids, beauvericin, lectins, triterpenes, pigments, benzofurans, $\alpha$-glucans, carotenoid pigments, and phenolic compounds [24,25]. Unlike other polypores, the Laetiporus sulphureus has a long history of consumption especially in North America, Japan and Thailand where it is considered a delicacy. Moreover, this fungus has long been used in Asian herbal medicine and is also known as a source of antitumor, antiviral, antiinflammatory, anticoagulant, antioxidant, antibacterial, cytostatic, and immunostimulative agents and a producer of HIV-1 reverse transcriptase inhibitors [26].
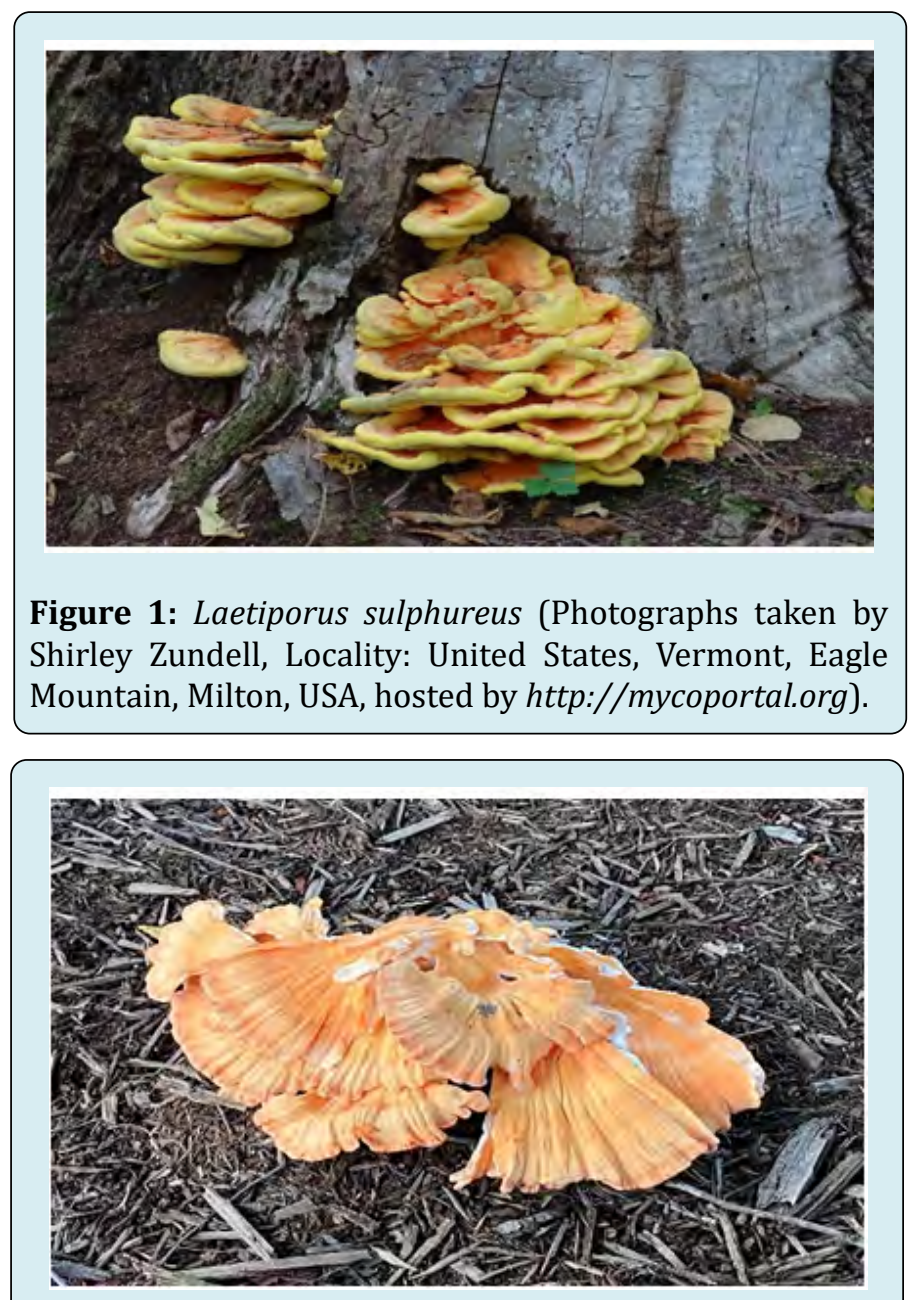

Figure 2: Laetiporus sulphureus (Photographs taken by Jillian Mattern, Locality: USA, Mississippi, Marshall, Wall Doxey State Park, hosted by http://mycoportal.org). 


\section{Open Access Journal of Microbiology \& Biotechnology}

\section{Antioxidant and Antimicrobial Activities of Laetiporus Sulphureus}

Due to their long history of medical use, the biologically active compounds and extracts from Laetiporus sulphureus exhibit a broad spectrum of pharmacological activities. Therefore, it can be easily assumed that presence of this mushroom into our lifestyle may benefit us. The numerous studies have shown that Laetiporus sulphureus nutritionally provides various key components such as carbohydrate, essential amino acids and fatty acids, vitamins, minerals, and fibre. Besides, the extracts prepared from fruiting bodies or mycelia of this mushroom have exhibited a number of medicinal properties such as immunomodulation, antitumor, anti-inflammatory, antioxidant, antimicrobial, and antihyperglysemic activities because of their biologically active components such as phenolics, triterpenes, and polysaccharides. Laetiporus sulphureus is also a suitable candidate to be used as a natural food preserving source [27].

Laetiporus sulphureus delivers bioactive ingredients that possess health benefits. About $75 \%$ of Laetiporus sulphureus secondary metabolites are composed of triterpenoids [28]. Laetiporus sulphureus can also be cultivated on a larger scale in a laboratory both as fruiting bodies in a solid-state surface culture and as a hyphal mycelium in a fermented submerged culture [29]. Zhang, et al. reported that twelve compounds were found in the mycelia extracts of Laetiporus sulphureus: oleic acid, palmitic acid, glycerol $\alpha$-monopalmitate, ergosterol, lactic acid, glycerol, succinate, uracil, D-mannitol, ring-(proline-glycine), cyclo-L-HypGly, and L-methyl L-glutamate, all showed antioxidant and antimicrobial activities [30-33]. Petrović, et al. reported that Laetiporus sulphureus is a rich source of carbohydrates and proteins. Mannitol and trehalose were the main free sugars, also the polyunsaturated fatty acids a-, g- and d-tocopherols were found. Oxalic and citric acids were the most abundant organic acids; cinnamic and p-hydroxybenzoic acids were quantified in the methanolic extract and could be related to the antioxidant properties. It was the polysaccharidic extract that exhibited higher antioxidant and antimicrobial activities, indicating that the compounds present in Laetiporus sulphureus possess stronger bioactivity [22,24].

Laetiporus sulphureus, hot-water extractable polysaccharides (H-SMPS) and enzymatic-extractable polysaccharides (E-SMPS) were successfully isolated from L. sulphureus. Both H-SMPS and E-SMPS were found to have high reducing power and potential scavenging activities against hydroxyl, DPPH, and superoxide anion radicals [34]. Antioxidant and antimicrobial potentials of Laetiporus sulphureus alcoholic extracts from dried fruiting bodies, dried mycelia broth and mycelia-free broth submerged cultures were investigated. For determination of potential antioxidant activity of the dried fruiting bodies and mycelia-free broth methanol extracts, the total phenols amount and scavenging capacity on 2,2-diphenyl1-picrylhydrazyl (DPPH) radicals have been analyzed. The results showed that the highest total phenols amount was found in fruiting bodies extract. Radical scavenging activity was found higher for fruiting bodies followed by mycelia-free broth extract. The antimicrobial effects of the ethanol extracts were analyzed against Candida albicans ATCC10321, Candida parapsilopsis CBS604, Escherichia coli ATCC8739, Staphylococcus aureus ATCC6538, Enterococcus faecalis and Staphylococcus epidermidis ATCC12228. Two extracts presented a wide antimicrobial spectrum and were active against both yeast and bacteria tested: fruit bodies extract and dried biomass extract $[35,36]$. Cyclohexane, dichloromethane, and methanol extracts of the medicinal mushroom Laetiporus sulphureus were tested for their antimicrobial, cytotoxic, and radical scavenging activities. All extracts resulted in the removal of $50 \%$ of the DPPH radicals. The average minimum inhibitory concentrations (MICs) against a select panel of microorganisms were between 125 and $250 \mathrm{~m} \mu \mathrm{g} / \mathrm{mL}$, and the methanolic extract significantly inhibited the growth of Staphylococcus aureus (MIC, 31.25 $\mathrm{m} \mu \mathrm{g} / \mathrm{mL}$ ). Also, cyclohexane and dichloromethane extracts inhibited the growth of Helicobacter pylori (MIC, $62.5 \mathrm{~m} \mu$ $\mathrm{g} / \mathrm{mL}$ ). Cyclohexane and dichloromethane extracts showed higher antiproliferative action compared with the methanol extract [37].

Patocka J, et al. [38] suggested that Laetiporus species could be used as supplementary agents in treatment of various diseases in addition to their nutritional properties and they would occupy an important place in the development of new pharmacological new agents due to their active components. The potential of Laetiporus species for use in design of modern medicines by determination of their phytochemical content and isolation of these compounds is noteworthy.

\section{Schizophyllum Commune}

Schizophyllum commune is a species of fungus in the genus Schizophyllum. The mushroom resembles undulating waves of tightly packed corals or loose Chinese fan. "Gillies" or "split gills" vary from creamy yellow to pale white in colour as illustrated in, Figures $3 \& 4$. The caps are $1-4 \mathrm{~cm}$ wide with white or grayish hairs. They grow in shelf-life arrangements, without stalks [39]. The gills, which produce basidiospores on their surface, split when the mushroom dries out, earning this mushroom the common name split gill. It is common in rotting wood [40]. It is the only known fungus capable of retracting by movement [41]. This mushroom is found throughout the world except Antarctica, where there is no wood to be used as a substrate. It is found in the wild on decaying trees after rainy seasons followed by dry spell where the mushrooms 


\section{Open Access Journal of Microbiology \& Biotechnology}

are naturally collected. The scientific classification of Schizophyllum commune was kingdom Fungi, Division Basidiomycota, Class Agaricomycetes, Order Agaricales, Family Schizophyllaceae, Genus Schizophyllum, species $S$. commune. Schizophyllum commune has been the subject of genetic analysis since the early twentieth century, when Kniep first described its tetrapolar pattern of sexuality. It has been utilized as a model system for studying mating-type gene function and mushroom development. In addition, the biochemistry and enzymology of the cell walls of S. commune has been a long-term focus of research. It belongs to the group of gilled mushrooms that includes the commercially valuable species Agaricus bisporus (white button mushroom) and Pleurotus ostreatus (oyster mushroom) [42].

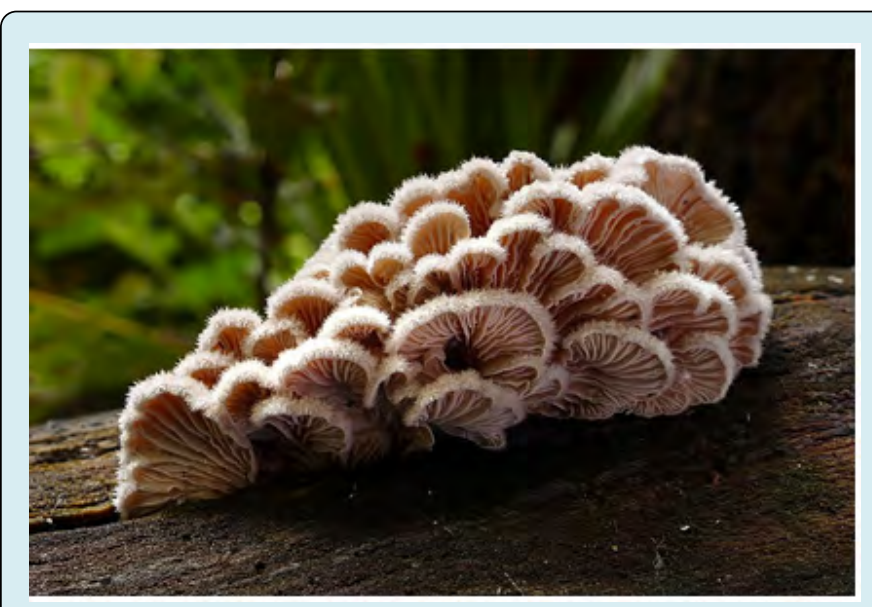

Figure 3: Schizophyllum commune (Photo was taken by Bernard Spragg, Locality: from Christchurch, New Zealand. Cited in https://en.wikipedia.org/wiki/Schizophyllum commune).

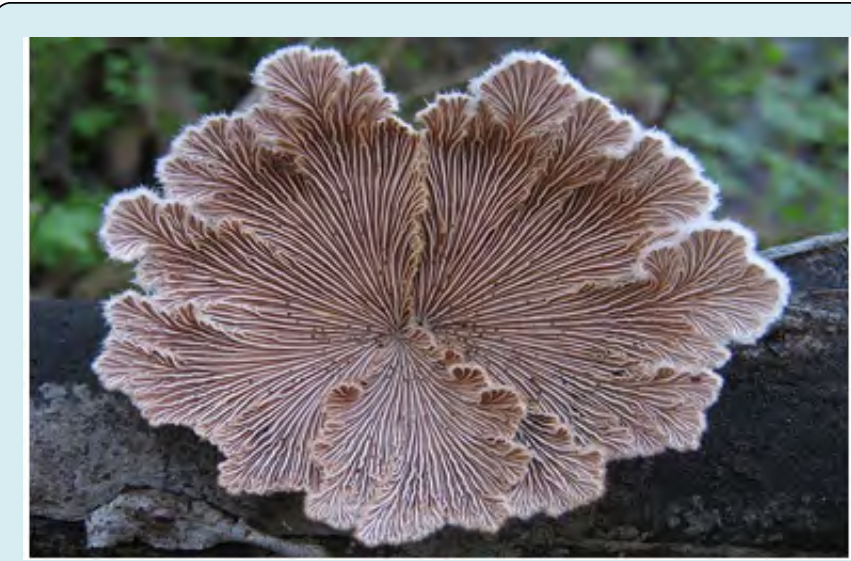

Figure 4: Schizophyllum commune (Locality: Marteleira, Lourinhã, Portugal. (Cited in https://commons.wikimedia. org/wiki/File:Schizophyllum_commune_Fr_299074.jpg).

\section{Schizophyllum Commune as Therapeutic Food}

Wild mushrooms with their delicate flavour and texture are recognized as a nutritious food and an important source of biologically active compounds with medicinal values. Generally, mushrooms are low in energy and high in dietary fibre [43], and an excellent source for antioxidants as they accumulate a variety of secondary metabolites, including phenolic compounds [44]. There are about 1200 species of mushroom used in 85 different countries for their gastronomic value and/or medicinal properties [45]. On the other hand, edible wild mushrooms are often regarded as being nutritionally high and with potential economic value. Many species with medicinal value are widely used in traditional medicine for a broad range of diseases [46]. Some species are regarded as therapeutic food for their anticarcinogenic, anti-cholesterolemic and anti-viral properties. Different medicinal (anticarcinogenic, anticholesterol, immunostimulating) effects of Schizophyllum commune are known but little is known about its nutritive values [47]. Edible wild mushrooms are often regarded as being nutritionally high and with potential economic value. Many species with medicinal value are widely used in traditional medicine for a broad range of diseases [46]. Some species like Schizophyllum commune are regarded as therapeutic food for their anti-carcinogenic, anti-cholesterolemic and anti-viral properties.

Most of the medicinal extracts from mushrooms Schizophyllum commune are different forms of polysaccharides and all of them are strengthens of the immune system with little or no side effects. Recent studies on various white rot fungi possess a number of beneficial medicinal properties such as antitumor, immune-modulators, anti-genotoxic, anti-oxidant, anti-inflammatory, anti-allergic, hypocholesterolemic, antihypertensive, anti-hyperglycemic, antimicrobial and antiviral activities. These activities have been reported for varies extracts and isolated compounds, such as polysaccharides, polysaccharide-protein complexes, proteoglycans, protein and DNA from oyster mushroom fermentation broth, mycelia or fruiting bodies. In particular, polysaccharides appear to be potent antitumor and immunemodulating substances, besides possessing other beneficial activities (Wound healing, anti-coagulate anti-nematode and others) [48-53].

\section{Conclusion}

In conclusion, it was considered that the use of Laetiporus sulphureus and Schizophyllum commune in complementary medicine is very important and urgent needed and these mushrooms have a significant potential as important natural agents in development of pharmacological drugs. 


\section{Open Access Journal of Microbiology \& Biotechnology}

\section{References}

1. Elkhateeb WA, Daba GM, Thomas PW, Wen TC (2019) Medicinal mushrooms as a new source of natural therapeutic bioactive compounds. Egypt Pharmaceu J 18(2): 88-101.

2. Glamočlija J, Sokovic M (2017) Fungi an source with huge potential for mushroom pharmaceuticals. Lekovite sirovine 37: 50-56.

3. Sharma D, Singh VP, Singh NK (2018) A Review on Phytochemistry and Pharmacology of Medicinal as well as Poisonous Mushrooms. Mini Rev Med Chem 18(13): 1095-1109?

4. El Hagrassi A, Daba G, Elkhateeb W, Ahmed E, El Dein AN, et al. (2020) In vitro bioactive potential and chemical analysis of the n-hexane extract of the medicinal mushroom, Cordyceps militaris. Malays J Microbiol 16(1): 40-48.

5. Elkhateeb WA, Daba GM, El Dein AN, Sheir DH, Fayad W, et al. (2020) Insights into the in-vitro hypocholesterolemic, antioxidant, antirotavirus, and anticolon cancer activities of the methanolic extracts of a Japanese lichen, Candelariella vitellina, and a Japanese mushroom, Ganoderma applanatum. Egyptian Pharmaceutical Journal 19(1): 67-73?

6. Elkhateeb WA, Elnahas MO, Thomas PW, Daba GM (2020) Trametes Versicolor and Dictyophora Indusiata Champions of Medicinal Mushrooms. Open Access Journal of Pharmaceutical Research 4(1): 1-7.

7. Elkhateeb WA, Daba G (2020) The endless nutritional and pharmaceutical benefits of the Himalayangold, Cordyceps; Current knowledge and prospective potentials. Asian Journal of Natural Product Biochemistry 18(2): 1-10.

8. Daba GM, Elkhateeb W, ELDien AN, Fadl E, El Hagrassi AM, et al. (2020) Therapeutic potentials of n-hexane extracts of the three medicinal mushrooms regarding their anticolon cancer, antioxidant, and hypocholesterolemic capabilities. Biodiversitas 21(6): 1-10.7

9. Thomas PW, Elkhateeb WA, Daba GM (2020) Chaga (Inonotus obliquus): a medical marvel but a conservation dilemma? Sydowia 72: 123-130.

10. Hernández RL, Franco GAC, Parra SJM, Domínguez MF (2008) Review of agricultural and medicinal applications of basidiomycete mushrooms. Tecnociencia Chihuahua 2(2): 95-107?

11. Rahi DK, RajakRC, Shukla KK, Pandey AK (2005) Diversity and nutraceutical potential of wild edible mushrooms of Central India. Microbial Diversity: Current Perspectives and Potential Applications, pp: 967-980.

12. Wasser SP (2014) Medicinal mushroom science: Current perspectives, advances, evidences, and challenges. Biomed J 37(6): 345-356.

13. Rahi DK, Malik D (2016) Diversity of mushrooms and their metabolites of nutraceutical and therapeutic significance. Journal of Mycology 1-18.

14. Elkhateeb WA, Daba GM, Elnahas M, Thomas P, Emam M (2020) Metabolic profile and skin-related bioactivities of Cerioporus squamosus hydromethanolic extract. Biodiversitas J Biological Div 21(10)!

15. Elkhateeb WA, Daba GM (2020) Termitomyces Marvel Medicinal Mushroom Having a Unique Life Cycle. Open Access Journal of Pharmaceutical Research 4(1): 1-4.

16. Elkhateeb WA (2020) What medicinal mushroom can do? Chem Res J 5(1): 106-118.

17. Barbosa MMB, Cruz RHSF, Calonge FD, Baseia IG (2014) Two new records of Cyathus species for South America. Mycosp 5(3): 425-428.

18. Elkhateeb WA, Daba GM, Elmahdy EM, Thomas PW, Wen TC, et al. (2019) Antiviral potential of mushrooms in the light of their biological active compounds. ARC J Pharmac Sci 5(2): 45-49?

19. Petrović J, Glamočlija J, Stojkovic DS, Ciric A, Nikolic M, et al. (2013) Laetiporus sulphureus, edible mushroom from Serbia: Investigation on volatile compounds, in vitro antimicrobial activity and in situ control of Aspergillus flavus in tomato paste. Food Chem Toxicol 59: 297-302.

20. Pleszczyńska M, Wiater A, Siwulski M, Szczodrak J (2013) Successful large-scale production of fruiting bodies of Laetiporus sulphureus (Bull: Fr.) Murrill on an artificial substrate. World Journal of Microbiol Biotechnol 29(4): 753-758.

21. Petrovic J, Papandreou M, Glamoclija J, Ciric A, Baskakis C, et al. (2014) Different extraction methodologies and their influence on the bioactivity of the wild edible mushroom Laetiporus sulphureus (Bull.) Murrill. Food \& Function 5(11): 2948-2960?

22. Alves MJ, Ferreira IC, Dias J, Teixeira V, Martins A, et al. (2012) A review on antimicrobial activity of mushroom (Basidiomycetes) extracts and isolated compounds. Planta Med 78(16): 1707-1718.

23. Sinanoglou VJ, Zoumpoulakis P, Heropoulos G, Proestos 


\section{Open Access Journal of Microbiology \& Biotechnology}

C, Ciric A, et al. (2015) Lipid and fatty acid profile of the edible fungus Laetiporus sulphurous. Antifungal and antibacterial properties. J Food Sci Technol 52(6): 32643272?

24. Bulam S, Ustun NS, Pekșen A (2019) Nutraceutical and Food Preserving Importance of Laetiporus sulphureus. Turkish Journal of Agriculture-Food Science and Technology 7(supp1): 94-100.

25. KhatuaS, Ghosh S, Acharya K(2017) Laetiporussulphureus (Bull: Fr.) Murr. as food as medicine. Pharmacognosy Journal 9(6): S1-S15.

26. Davoli P, Mucci A, Schenetti L, Weber RW (2005) Laetiporic acids, a family of non-carotenoid polyene pigments from fruit bodies and liquid cultures of Laetiporus sulphureus (Polyporales, Fungi). Phytochemistry 66(7): 817-823.

27. Zhang J, Lv J, Zhao L, Shui X, Wang LA (2018) Antioxidant and antimicrobial activities and chemical composition of submerged cultivated mycelia of Laetiporus sulphureus. Chemistry of Natural Compounds 54(6): 1187-1188.

28. Sułkowska Ziaja K, Muszyńska B, Gawalska A, Sałaciak K (2018) Laetiporus sulphureus-chemical composition and medicinal value. Acta Scientiarum Polonorum Hortorum Cultus 17(1): 87-96.

29. Klaus A, Kozarski M, Niksic M, Jakovljevic D, Todorovic N, et al. (2013) The edible mushroom Laetiporus sulphureus as potential source of natural antioxidants. Int J Food Sci Nutr 64(5): 599-610.

30. Smith AH, Weber NS (1980) The mushroom hunter's field guide. University of Michigan Press, USA.

31. Zhao H, Lan Y, Liu H, Zhu Y, Liu W, etal. (2017) Antioxidant and hepatoprotective activities of polysaccharides from spent mushroom substrates (Laetiporus sulphureus) in acute alcohol-induced mice. Oxid Med Cell Longev pp: 5863523.

32. Popa G, Cornea CP, Luta G, Gherghina E, Bubueanu C, et al. (2016) Antioxidant and antimicrobial properties of Laetiporus sulphureus (Bull.) Murrill. AgroLife Scientific Journal 5(1): 168-173?

33. Kolundzic MD, Grozdanic NO, Stanojkovic TP, Milenkovic MT, Dinic MR, et al. (2016) Antimicrobial and Cytotoxic Activities of the Sulphur Shelf Medicinal Mushroom, Laetiporus sulphureus (Agaricomycetes), from Serbia. International Journal of Medicinal Mushrooms 18(6): 469-476?

34. Patocka J (2019) Will the sulphur polypore (Laetiporus sulphureus) become a new functional food? Global Journal of Medical and Clinical Case Reports 6(1): 006009.'

35. Davis RM, Sommer R, Menge JA (2012) Field Guide to Mushrooms of Western North America. $1^{\text {st }}$ (Edn.), Berkeley: University of California Press, USA, pp: 472.

36. Guarro J, Genéj Ams (1999) Developments in Fungal Taxonomy. Clin Microbiol Rev 12(3): 454-500.

37. Kuo M (2003) Schizophyllum commune. Mushroom Expert.

38. Eastwood DC (2014) Evolution of fungal wood decay. In Deterioration and Protection of Sustainable Biomaterials. American Chemical Society, pp: 93-112.

39. Emilia B, Grazyna J, Zofia L (2006) Edible mushroom as a source of valuable nutritive constituents. Ac Sci Pol Technol Ali 5(1): 5-20.

40. Cheung LM, Cheung PCK, Ooi VEC (2003) Antioxidant activity and total phenolics of edible mushroom extracts. Food Chem 81(2): 249-255.

41. De Roman M, Boa E, Woodward S (2006) Wild gathered fungi for health and rural livelihoods. Proc Nutr Soc 65(2): 190-197.

42. Nakano T, Oka K, Hanba K, Morita S (1996) Intratumoral administration of sizofiran activates Langerhans cells and T-cell infiltration in cervical cancer. J Clin Immunol Immunopathol 79(1): 79-86.

43. Khan A, Rahman MM, Tania M, Ahmed S, Uddin N (2011) Pleurotus sajor-cajo and Pleurotus florida mushrooms improve some extent of the antioxidant systems in the liver of hypercholesterolemia rats. The open Nutraceuticals J 4: 20-24.

44. Zhao S, Gao Q, Rong C, Wang S, Zhao Z, et al. (2020) Immunomodulatory effects of edible and medicinal mushrooms and their bioactive immunoregulatory products. J Fungi 6(4): 269.

45. Hobbs C, Beinfield H (2002) Medicinal mushrooms: an exploration of tradition, healing, and culture. Botanica Press.'

46. Motta F, Gershwin ME, Selmi C (2021) Mushrooms and immunity. Journal of autoimmunity 117: 102576?

47. Badalyan S, Barkhudaryan A, Rapior S (2019) Recent progress in research on the pharmacological potential of mushrooms and prospects for their clinical application. Medicinal Mushrooms, pp: 1-70. 


\section{Open Access Journal of Microbiology \& Biotechnology}

48. Elkhateeb WA, Elnahas MO, Thomas PW, Daba GM (2019) To Heal or Not to Heal? Medicinal Mushrooms Wound Healing Capacities. ARC Journal of Pharmaceutical Sciences 5(4): 28-35.

49. Elkhateeb WA, Daba GM, Elnahas MO, Thomas PW (2019) Anticoagulant capacities of some medicinal mushrooms. ARC J Pharma Sci 5: 12-16.

50. Elkhateeb W, Elnahas MO, Paul W, Daba GM (2020) Fomes fomentarius and Polyporus squamosus models of marvel medicinal mushrooms. Biomed Res Rev 3(1): 119.

51. Elkhateeb WA, Daba GM (2021) Mycotherapy of the good and the tasty medicinal mushrooms Lentinus, Pleurotus, and Tremella. Journal of Pharmaceutics and Pharmacology Research 4(2): 1-6.

52. Elkhateeb WA, Daba GM (2021) The Fascinating Bird's Nest Mushroom, Secondary Metabolites and Biological Activities.] International Journal of Pharma Research and Health Sciences 9 (1): 3265-3269.

53. Elkhateeb WA, Daba GM, Gaziea SM (2021) The AntiNemic Potential of Mushroom against Plant-Parasitic Nematodes. Open Access Journal of Microbiology \& Biotechnology 6(1): 1-6. 